\title{
$\begin{array}{ll}\text { Research Square } & \text { Preprints are preliminary reports that have not undergone peer review. } \\ \text { They should not be considered conclusive, used to inform clinical practice, } & \text { or referenced by the media as validated information. }\end{array}$
}

\section{Efficacy and Safety Among Second-Generation and Other Basal Insulins in Adult Patients with Type 1 Diabetes: A Systematic Review and Network Meta- Analysis}

\section{Martin -}

Osaka University: Osaka Daigaku

Yi Zhou

Osaka University: Osaka Daigaku

\section{Tatsuya Takagi}

Osaka University: Osaka Daigaku

Yu-Shi Tian ( $\nabla$ yushi-tian@phs.osaka-u.ac.jp )

Osaka University: Osaka Daigaku https://orcid.org/0000-0002-8988-9453

\section{Research Article}

Keywords: basal insulin, glycemic control, hypoglycemia, weight gain, type 1 diabetes, network metaanalysis

Posted Date: June 4th, 2021

DOI: https://doi.org/10.21203/rs.3.rs-556854/v1

License: (c) (1) This work is licensed under a Creative Commons Attribution 4.0 International License. Read Full License

Version of Record: A version of this preprint was published at Naunyn-Schmiedeberg's Archives of Pharmacology on July 28th, 2021. See the published version at https://doi.org/10.1007/s00210-02102128-9. 


\section{Abstract}

Aims: To assess the comparative efficacy and safety of second-generation basal insulins (glargine U300 and degludec U100) vs. neutral protamine Hagedorn (NPH) and first-generation basal insulins (glargine U100 and detemir) in type 1 diabetes (T1D) adults.

Methods: PubMed, the Cochrane Library, ClinicalTrials.gov, and Google Scholar (until January 2021) were systematically searched. Randomized controlled trials (RCTs) with $\geq 12$ weeks of follow-up comparing efficacy $\left(\mathrm{HbA}_{1 \mathrm{c}}\right)$ or safety (hypoglycemia and weight gain) between second-generation basal insulins vs. other basal insulins in T1D adults were included. Bayesian network meta-analyses were used to estimate risk ratio, hazard ratio, and mean difference. Grading of Recommendations, Assessment, Development and Evaluation (GRADE) was used to appraise evidence certainty.

Results: Eighteen RCTs ( $\geq 24$ weeks of follow-up) involving 7,283 randomized participants were included for main analysis. Moderate to high certainty evidence suggested that second-generation basal insulins showed equivalent $\mathrm{HbA}_{1 \mathrm{c}}$ reduction compared with $\mathrm{NPH}$ and first-generation basal insulins. Compared with second-generation basal insulins, low to high certainty evidence suggested that NPH was associated with a higher risk of patients experiencing severe hypoglycemia; NPH and first-generation basal insulins were associated with a higher rate of nocturnal confirmed hypoglycemic events. For the weight gain, glargine U300 was comparable to detemir (low certainty), but degludec U100 was greater than detemir (moderate certainty).

Conclusions: In T1D adults, second-generation basal insulins maintained equivalent efficacy of glycemic control (moderate to high certainty), with differences in safety (low to high certainty) compared with NPH and first-generation basal insulins during $\geq 24$ weeks of follow-up.

\section{Introduction}

Type 1 diabetes (T1D) is characterized by insulin deficiency and resultant hyperglycemia (DiMeglio et al. 2018). Patients with T1D require lifelong insulin administration, and basal insulin preparations are the most used and essential replacement to mimic pancreatic insulin secretion. Basal insulins, including human neutral protamine Hagedorn (NPH), first-generation analogs (i.e., glargine $100 \mathrm{U} / \mathrm{mL}$ [glargine U100] or detemir), and second-generation analogs (i.e., glargine $300 \mathrm{U} / \mathrm{mL}$ [glargine U300] or degludec), have been clinically used to control fasting glucose levels. However, since different preparations exhibit distinct pharmacokinetic and pharmacodynamics profiles, basal insulin preparations do not always provide the same efficacy and safety (Mathieu et al. 2017).

Systematical comparisons on the efficacy (glycemic controls) or safety (iatrogenic severe hypoglycemic events) between first-generation basal insulins vs. NPH have been discussed in an umbrella review (Laranjeira et al. 2018). Currently, there are no trials directly comparing the second-generation basal insulins with NPH or glargine U300 with detemir in T1D adults. The most recent updated network metaanalysis comparing NPH and detemir with the second-generation basal insulins in T1D adults indicated 
similar reductions in $\mathrm{HbA}_{1 \mathrm{c}}$ and severe hypoglycemic events (Bain et al. 2020). However, their network meta-analysis on safety only concerned with severe hypoglycemia, defined as the most dangerous level (level one) by American Diabetes Association (ADA) (International Hypoglycaemia Study Group 2017). In contrast, the confirmed hypoglycemia (level two) was not evaluated. The confirmed hypoglycemia reduces patient quality of life and will develop to severe one if not properly treated (International Hypoglycaemia Study Group 2017). Especially, the confirmed hypoglycemia occurring at night is also dangerous and should also be aware. In their outcome of severe hypoglycemia, only the number of events per person-year was updated. The synthesized results based on the number of patients who experience hypoglycemia will provide a different aspect of information and is of clinical importance. On the other hand, insulin users often report body weight gains, limiting insulin intensification or basal-bolus therapy. Body weight gain caused by insulin-induced lipogenesis will increase cardiovascular risk (Purnell et al. 1998; Russell-Jones and Khan 2007). As one of the important adverse events, body weight gain was not included in their analysis. Furthermore, the Grading of Recommendations, Assessment, Development and Evaluation (GRADE) certainty of evidence was also unavailable in either efficacy or safety outcome. Thus, a more comprehensive analysis of the efficacy and safety of second-generation basal insulins is still necessary.

This study systematically evaluates the efficacy and safety of second-generation insulins analogs vs. $\mathrm{NPH}$ or first-generation basal insulins in T1D adults using the Bayesian network meta-analysis.

\section{Methods}

This systematic review and network meta-analysis was reported as per the Preferred Reporting Items for Systematic Review and Meta-Analysis (PRISMA) statement (Table S1) and was performed according to the AMSTAR 2 critical appraisal tool (Moher et al. 2009; Hutton et al. 2015; Shea et al. 2017). In addition, the protocol has been registered in the International Prospective Register of Systematic Reviews (PROSPERO) database (CRD42020175051).

\section{Data sources and searches}

Two investigators ( $M$ and $Y Z$ ) systematically searched PubMed, the Cochrane Library, ClinicalTrials.gov, and Google Scholar from inception until 12 January 2021 (Table S2). The study selection process was recorded using a PRISMA-compliant flow chart (Moher et al. 2009). In addition, reference lists of relevant articles were reviewed for further study search.

\section{Study selection}

The eligible studies were limited to 1) randomized controlled trials (RCTs) (study design); 2) adults with T1D (aged $\geq 18$ years) and $\geq 12$ weeks of follow-up under basal and mealtime insulins (study population); 3) interventions and comparisons were second-generation basal insulins vs. NPH, glargine $\mathrm{U} 100$, or detemir; 4) studies reported at least one of the following outcomes: change in $\mathrm{HbA}_{1 \mathrm{c}}$ or body weight between baseline and endpoint, severe hypoglycemia, nocturnal severe hypoglycemia, confirmed 
hypoglycemia, or nocturnal confirmed hypoglycemia; 5) in case of duplicate studies, we extracted data with the longest follow-up if retrievable. Severe hypoglycemia was defined as an event requiring assistance or as per the American Diabetes Association (ADA) categories (International Hypoglycaemia Study Group 2017). Confirmed hypoglycemia was defined as an event with plasma glucose concentration $<3.0-3.1 \mathrm{mmol} / \mathrm{L}(<54-56 \mathrm{mg} / \mathrm{dL}$ ) or severe hypoglycemia. Nocturnal hypoglycemia was defined as an event occurring during the night (23.00 to 06.00 hours or narrower duration). The exclusion criteria included participants with recurrent severe hypoglycemia, cross-over trials with $<12$ weeks of follow-up for each period, and children, adolescents, pregnant or breastfeeding women.

\section{Data extraction and quality assessment}

Two investigators ( $M$ and $Y Z$ ) independently extracted data from the eligible RCTs and evaluated the qualities of the eligible RCTs and the network evidence. A third investigator (YST) resolved disagreements. Extracted data were comprised of study characteristics and outcomes. Outcomes were comprised of the following: 1) changes in $\mathrm{HbA}_{1 \mathrm{c}}(\%)$ and mean body weight $(\mathrm{kg})$ between baseline and endpoint; 2) the number of patients experiencing severe hypoglycemia, nocturnal severe hypoglycemia, confirmed hypoglycemia, and nocturnal confirmed hypoglycemia (percentage of participants experiencing $\geq$ one hypoglycemic event); and 3 ) the number of events on severe hypoglycemia, nocturnal severe hypoglycemia, confirmed hypoglycemia, and nocturnal confirmed hypoglycemia (hypoglycemic events per person-year). Person-year data were extracted either from the trial results or by multiplying the sample size by the follow-up years. If the SE or SD of the change was unable to be extracted, we imputed it according to the Cochrane guidelines (Higgins et al. 2019a) or using the average SD of the change reported from similar trials with the same interventions.

The risk of bias was assessed using the Cochrane risk of bias (RoB) 2.0 tool. Ratings of risk of bias within individual trials comprised the randomization process, deviations from intended interventions, missing outcome data, measurement of the outcome, selection of the reported result, and overall bias (Higgins et al. 2019b). Evidence certainty was appraised using the GRADE approach (Puhan et al. 2014) and the rules in Confidence in Network Meta-Analysis (CINeMA) (Nikolakopoulou et al. 2020). Evidence certainty ratings comprised within-study bias, across-studies bias, indirectness, imprecision, heterogeneity, and incoherence.

\section{Data synthesis and statistical analysis}

The main analysis was measured in $\geq 24$ weeks of follow-up. Sensitivity analysis was measured in $\geq 12$ weeks of follow-up to explore the robustness of effect sizes. The effect sizes were comprised of mean difference (MD) for assessing the changes in $\mathrm{HbA}_{1 \mathrm{c}}$ or body weight; risk ratio (RR) for assessing the risk on the number of patients experiencing hypoglycemia; and hazard ratio (HR) for assessing the number of hypoglycemic events per person-year. We assumed exchangeability between treatments (direct and indirect evidence concurs (van Valkenhoef et al. 2012)) and used a random-effects model to allow for inevitable heterogeneity (Higgins et al. 2012). The effect sizes were calculated using the Markov Chain Monte Carlo (MCMC) simulation in the Bayesian hierarchical model (BHM). We used the binomial 
distribution with a log link function to calculate RR, the Poisson distribution with a log link function to calculate HR, and the normal distribution with an identity link function to calculate MD (Dias et al. 2013a). We set a uniform prior distribution model, discarded the first 50,000 iterations, and performed 100,000 MCMC simulations with a thinning of 20 (5,000 samples) for four chains.

Heterogeneity in the treatment comparators was assessed using per-comparison $R$ (van Valkenhoef 2014). Assessment of inconsistency between direct and indirect evidence was analyzed by node splitting method (Dias et al. 2013b). The risk of bias across trials (publication bias) was assessed using comparison-adjusted funnel plots (Chaimani and Salanti 2012). Funnel plot asymmetry was tested using Egger's regression (Egger et al. 1997). Statistical significance was defined as two-sided with $a<0.05$. The funnel plots and Egger's regression were performed using the "netmeta" (version 1.2-0) package. Network meta-analyses, with statistical significance defined as $95 \%$ credible interval (Crl) did not cross 0.00 (MD) or 1.00 (RR and HR), were performed using the "gemtc" (version 0.8-2) package implemented in R (version 4.0.2). The $2.5 \%$ and $97.5 \%$ quantiles of the Crl were derived from the Bayesian inference.

\section{Results}

\section{Search results}

The literature search yielded 4,965 citations (Fig. 1). Among them, we reviewed 50 full-text articles and finally included 31 eligible RCTs published between 2000 and 2019 in this network meta-analysis (Table S3).

\section{Study selection and characteristics}

The total of 31 RCTs included 10,229 randomized participants with follow-up durations ranging from 12 to 104 weeks. The detailed characteristics were summarized in Table S4. Eighteen trials $(N=7,283)$ with $\geq 24$ weeks of follow-up were included for the main analysis. Network graphs of all outcomes for main analysis were shown in Fig. 2. The mean age across the RCTs ranged from 34 to 48.2 years. Most trials were multicenter and received financial aid.

Overall risk of bias within trials was that one trial (3\%) had a low risk of bias, twenty (65\%) had some concerns, and ten (32\%) had a high risk of bias (Fig. S1). Table S5 shows the Cochrane risk of bias judgments. Table 66 shows the extracted data for each outcome.

\section{Comparisons of outcomes}

\section{Efficacy outcome-change in $\mathrm{HbA}_{1 \mathrm{c}}$}

We conducted a network meta-analysis on change in $\mathrm{HbA}_{1 \mathrm{c}}$ that included $18 \mathrm{RCTs}$ (Fig. 2a). The MD of $\mathrm{HbA}_{1 \mathrm{c}}$ from baseline to $\geq 24$ weeks between the second-generation basal insulins vs. NPH or the firstgeneration basal insulins did not show significant differences (Fig. 3). 


\section{Safety outcome-number of patients experiencing hypoglycemia}

Severe, confirmed, and nocturnal confirmed hypoglycemia were evaluated by three network metaanalyses, including 14, 8 , and 8 RCTs on RR, respectively (Fig. 2c-2e). A significantly greater number of patients experiencing severe hypoglycemia were shown in the NPH group compared with the degludec U100 group (RR 1.88 [95\% Crl 1.30 to 2.71]) and the glargine U300 group (RR 1.98 [95\% Crl 1.08 to 3.68]) (Fig. 4). No other statistical differences existed in second and first-generation basal insulins for the number of patients experiencing severe hypoglycemia (Fig. 4). Furthermore, there was no significant difference among comparisons for the number of patients experiencing confirmed and nocturnal confirmed hypoglycaemic events (Fig. 4). Unfortunately, the RR of nocturnal severe hypoglycemia could not be determined due to the lack of data.

\section{Safety outcome-number of hypoglycemic events per person-year}

Four network meta-analyses on HR of severe, nocturnal severe, confirmed, and nocturnal confirmed hypoglycemic events were conducted, including 14, 9, 8, and 9 RCTs, respectively (Fig. 2f-2i). For severe, nocturnal severe, and confirmed hypoglycemia, NPH showed a greater number of hypoglycemic events per person-year than degludec U100 (HR 2.03 [95\% Crl 0.98 to 4.07], HR 3.16 [95\% Crl 0.58 to 14.90], HR 1.41 [95\% Crl 0.99 to 1.98], respectively) (Fig. 5). However, no statistical significance was detected. There were also no significant differences for other comparisons between glargine U300 and NPH or the firstgeneration basal insulins (Fig. 5). For nocturnal confirmed hypoglycemic events, compared with the second-generation basal insulins, NPH and the first-generation basal insulins showed greater event numbers per person-year [NPH vs. degludec U100 (HR 2.84 [95\% Crl 1.88 to 4.38]), glargine U100 vs. degludec U100 (1.35 [95\% Crl 1.12 to 1.63]), detemir vs. degludec U100 (HR 1.55 [95\% Crl 1.25 to 1.95]), NPH vs. glargine U300 (HR 2.78 [95\% Crl 1.69 to 4.68]), glargine U100 vs. glargine U300 (HR 1.32 [95\% Crl 1.03 to 1.74]), and detemir vs. glargine U300 (HR 1.52 [95\% Crl 1.08 to 2.22])] (Fig. 5).

\section{Safety outcome-weight gain}

We conducted a network meta-analysis on the MD of change in body weight, including 14 RCTs (Fig. 2b). Compared with degludec U100, detemir resulted in less weight gain (MD 0.60 [95\% Crl-1.13 to - 0.01]) significantly. However, no other significant differences were found within comparisons (Fig. 6).

\section{Quality of evidence network (GRADE), heterogeneity, and publication bias}

The GRADE assessment rules of network estimates were presented in Table S7. The GRADE confidence ratings for each network were incorporated in Fig. 2-5. Among the GRADE domains, major and some concerns were mainly related to imprecision and within-study bias (Fig. S2). Incoherence analyses did not apply to some pairwise comparisons owing to the absence of either direct or indirect evidence (Fig. S2). A 
comparison-adjusted funnel plot and Egger's regression test did not found asymmetry in all outcomes (Fig. S3). We observed heterogeneity on some pairwise comparisons (Fig. S4) but found no evidence of inconsistency from node splitting results (Table S8).

\section{Sensitivity analysis}

We conducted a sensitivity analysis to test the robustness of the effect sizes of all outcomes by adding thirteen $\geq 12$-week follow-up eligible trials (Table S6). Changes in $\mathrm{HbA}_{1 \mathrm{c}}$ and body weight outcomes showed robust results (Fig. S5a). Degludec U100 and glargine U300 showed the same effect sizes for the number of patients with severe hypoglycemia. The statistically significant difference between degludec U100 and NPH was robust. However, the statistically significant difference between glargine U300 and $\mathrm{NPH}$ was not maintained. Both degludec U100 and glargine U300 showed statistically lower numbers of patients on nocturnal confirmed hypoglycemia, which differed from the main analysis (Fig. S5b). For the HR of confirmed hypoglycemic events (Fig. S5c), degludec U100 vs. NPH showed a significant difference, while glargine U300 vs. NPH remained insignificant. The HR of the nocturnal confirmed hypoglycemic events produced larger effect sizes and robust significances (Fig. S5c).

\section{Discussion}

The present systematic review and network meta-analyses provide an evidence-based comparison of efficacy and safety between second-generation basal insulins vs. NPH or first-generation basal insulins for T1D adults. The efficacy evaluated in terms of the change in $\mathrm{HbA}_{1 \mathrm{c}}$ did not show significant differences between the second-generation basal insulins vs. NPH or first-generation basal insulins. In addition, sensitivity analysis showed this result is consistent in follow-up durations from either $\geq 24$ weeks or $\geq 12$ weeks.

This result of efficacy is consistent with Bain et al. (2020) that concluded no significant differences among insulin analogs in T1D adults (Bain et al. 2020). However, this result is inconsistent with another network meta-analysis (Tricco et al. 2021) that showed second-generation basal insulin degludec was superior to NPH in reducing $\mathrm{HbA}_{1 \mathrm{c}}$. The inconsistent results between Tricco et al. (2021) and our study arised in the inclusion criteria. We only focused on RCTs for T1D adults (aged $\geq 18$ years) with $\geq 24$ or $\geq$ 12 weeks of follow-up, while Tricco et al. (2021) mixed observational studies, children, pregnant women with irrespective follow-up duration (8 days to 2 years).

Hypoglycemia has been considered to be the barrier to achieve stringent $\mathrm{HbA}_{1 \mathrm{c}}$ levels (Cryer 2008; Mathieu et al. 2017). In this study, we investigated level one and level two hypoglycemia (International Hypoglycaemia Study Group 2017), including nocturnal one, and analyzed safety in the number of patients experiencing hypoglycemia and in the number of hypoglycemic events per person-year to provide comprehensive safety information. This meta-analysis found that the second-generation basal insulins were associated with significantly fewer patients experiencing severe hypoglycemia than NPH, with certainty evidence from moderate to low. Furthermore, the number of nocturnal confirmed hypoglycemic events per person-year of the second-generation basal insulins was significantly lower than NPH and first- 
generation basal insulins, with certainty evidence from moderate to high. For other hypoglycemia outcomes, no significant differences were detected in terms of neither RR nor HR. The inconsistent results from RR and HR were obtained here due to different measures of the event numbers and whether considering the time-dependent unit. Therefore, both RR and HR are suggested to be taken into consideration for assessing the safety of basal insulins.

Severe hypoglycemia has been well documented as a life-threaten event. Nocturnal confirmed hypoglycemia that is mainly affected by basal insulins can be considered less aware owing to the night. However, nocturnal confirmed hypoglycemia affects the patient quality of life and has a risk of escalating to a severe level (International Hypoglycaemia Study Group 2017). Therefore, the benefits of the secondgeneration basal insulins can be addressed. Stable pharmacodynamic profiles of the second-generation basal insulins may be a major factor (Heise et al. 2012; Becker et al. 2014). However, the evidence levels were not strong. Therefore, attention should be paid to these results.

The NICE network meta-analysis in T1D adults (NG17) (NICE (National Institute for Health and Care Excellence) 2015) and its update found no difference among basal insulins in the rate of severe hypoglycemic events (Dawoud et al. 2018; Bain et al. 2020). Our results are in line with these reports. However, based on the number of patients experiencing severe hypoglycemia, we found differences between second-generation basal insulins and NPH. Compared with Tricco et al. (2021), our result differed in detecting the statistical differences in the number of patients experiencing nocturnal hypoglycemia. Tricco et al. (2021) showed that degludec was statistically superior to NPH in the incidence of nocturnal hypoglycemia. However, we only detected differences in the rate of nocturnal confirmed hypoglycemia events. The differences may arise from the different inclusion criteria. Furthermore, we found that they included a study reported by Pieber et al. (Pieber et al. 2007), which was argued to have apparent inherent biases (Alcolado et al. 2008) and thus was excluded in this study. Compared to direct meta-analyses, we found a consistent difference that second-generation basal insulins had a lower rate of nocturnal hypoglycemic events than first-generation glargine U100 (Ratner et al. 2013; Vora et al. 2014; Liu et al. 2018; Díez-Fernández et al. 2019).

Basal insulin therapy can commonly cause weight gain in T1D. Weight gain negatively alters lipid levels and blood pressure, thereby increasing the risk of cardiovascular disease (Purnell et al. 1998; RussellJones and Khan 2007). Weight gain also affects the waist-to-hip ratio (Purnell et al. 1998), which may affect patients' self-perception, especially young people, leading to psychological insulin resistance (Brod et al. 2009). Our results showed no differences between second-generation basal insulins and NPH or first-generation basal insulins, except for the comparison between detemir and degludec U100. Detemir significantly resulted in less weight gain than degludec U100 with moderate certainty evidence. Therefore, when tailoring the treatment concerning weight gain, detemir and glargine U300 could be selected.

The strength of this study is the new insights into the harms concerning the second-generation basal insulins vs. other basal insulins in T1D adults by evaluating different categories of hypoglycemia in detail and changes in body weight. Meanwhile, we updated the results of the efficacy. The current network 
meta-analysis applied the GRADE approach and used rigorous methodology by adhering to the AMSTAR 2.0 critical appraisal tool and the PRISMA and Cochrane's guidelines. In addition, we quantitively evaluated the certainty evidence of each comparison. The information provided here may help select an appropriate basal insulin type for T1D adult patients under different backgrounds, which could be considered as a clinical imperative.

Several limitations should be considered when interpreting the results. First, the quality of RCTs in adults enhanced the information accuracy here; however, information may be limited. Second, due to the lack of information from RCT, we did not adjust the potential confounders, such as bolus (meal) insulin, frequency, injection device-dependent, and newly initiating effects on the efficacy and safety. Third, the trials involved in this network meta-analysis were limited in the number, varying from 8 to 14. Fourth, open-label design and sponsorship from pharmaceutical manufacturers in almost all included trials and differences in study design across trials. Finally, heterogeneity was observed for some comparisons, which may be accounted for different protocol designs. In the future, we expect more consolidated protocols and study designs in the RCTs and hopefully to update the present evidence.

In conclusion, based on the present eligible studies, second-generation basal insulins achieved similar $\mathrm{HbA}_{1 \mathrm{c}}$ levels compared with NPH and first-generation basal insulins with moderate to high certainty evidence. For hypoglycemia, second-generation basal insulins showed partial merits. For the weight gain, glargine U300 was comparable to detemir, while degludec U100 resulted in more weight gain than detemir. With the caution of the limitations of this study, the information can help tailor basal insulin for T1D adults via evidence-informed practice.

\section{Abbreviations}

ADA American Diabetes Association; BHM Bayesian hierarchical model; CINeMA Confidence in Network Meta-Analysis; Crl Credible interval; GRADE Grading of Recommendations, Assessment, Development and Evaluation; NICE National Institute for Health and Care Excellence; HR Hazard ratio; MCMC Markov Chain Monte Carlo; MD Mean difference; NPH neutral protamine Hagedorn; RR Risk ratio; T1D Type 1 diabetes

\section{Declarations}

\section{Ethics approval}

Not applicable.

\section{Consent to participate}

Not applicable.

\section{Consent for publication}

Not applicable. 


\section{Competing interests}

The authors declare no competing interests.

\section{Data availability}

The datasets used in this research are published in Supplemental Material (Table S6) which can be found in this published article.

\section{Author contributions}

$\mathrm{M}$ conceived and conceptualized the study. $\mathrm{M}$ and $\mathrm{YZ}$ designed the methodology and contributed to data curation, data analysis, interpretation of the results, discussion, and drafted the manuscript. YST contributed to data analysis and discussion. YST and TT reviewed the manuscript. All authors revised and approved the final manuscript. The authors declare that all data were generated in-house and that no paper mill was used.

\section{Funding}

This research received no specific grant from any funding agency in the public, commercial or not-forprofit sectors.

\section{Acknowledgments}

We would like to thank Dr. Mitsuyoshi Takahara (Department of Diabetes Care Medicine, Osaka University Graduate School of Medicine) and Prof. lichiro Shimomura (Department of Metabolic Medicine, Osaka University Graduate School of Medicine) for their comments.

\section{References}

1. Alcolado J, Poole CD, Peters JR, Currie CJ (2008) Potential flaws and biases in a randomized controlled trial (RCT) of insulin determir vs. insulin glargine by Pieber and colleagues. Diabet Med 25:115-116. https://doi.org/10.1111/j.1464-5491.2007.02335.x

2. Bain S, Feher M, Fisher M, et al (2020) A review of the NG17 recommendations for the use of basal insulin in type 1 diabetes. Diabet Med 37:219-228. https://doi.org/10.1111/dme.14180

3. Becker RHA, Dahmen R, Bergmann K, et al (2014) New Insulin Glargine 300 units·mL-1 Provides a More Even Activity Profile and Prolonged Glycemic Control at Steady State Compared With Insulin Glargine 100 units·mL-1. Diabetes Care 38:637-643. https://doi.org/10.2337/dc14-0006

4. Brod M, Kongs $\varnothing \mathrm{JH}$, Lessard S, Christensen TL (2009) Psychological insulin resistance: patient beliefs and implications for diabetes management. Qual Life Res 18:23. https://doi.org/10.1007/s11136-008-9419-1 
5. Chaimani A, Salanti G (2012) Using network meta-analysis to evaluate the existence of small-study effects in a network of interventions. Res Synth Methods 3:161-176. https://doi.org/10.1002/jrsm.57

6. Cryer PE (2008) The Barrier of Hypoglycemia in Diabetes. Diabetes 57:3169-3176. https://doi.org/10.2337/db08-1084

7. Dawoud D, O'Mahony R, Wonderling D, et al (2018) Basal insulin regimens for adults with type 1 diabetes mellitus: a systematic review and network meta-analysis. Value Heal 21:176-184. https://doi.org/10.1016/j.jval.2017.04.024

8. Dias S, Sutton AJ, Ades AE, Welton NJ (2013a) Evidence synthesis for decision making 2: a generalized linear modeling framework for pairwise and network meta-analysis of randomized controlled trials. Med Decis Mak 33:607-617. https://doi.org/10.1177/0272989X12458724

9. Dias S, Welton NJ, Sutton AJ, et al (2013b) Evidence synthesis for decision making 4: Inconsistency in networks of evidence based on randomized controlled trials. Med Decis Mak 33:641-656. https://doi.org/10.1177/0272989X12455847

10. Díez-Fernández A, Cavero-Redondo I, Moreno-Fernández J, et al (2019) Effectiveness of insulin glargine U-300 versus insulin glargine U-100 on nocturnal hypoglycemia and glycemic control in type 1 and type 2 diabetes: a systematic review and meta-analysis. Acta Diabetol 56:355-364. https://doi.org/10.1007/s00592-018-1258-0

11. DiMeglio LA, Evans-Molina C, Oram RA (2018) Type 1 diabetes. Lancet 391:2449-2462. https://doi.org/10.1016/S0140-6736(18)31320-5

12. Egger M, Smith GD, Schneider M, Minder C (1997) Bias in meta-analysis detected by a simple, graphical test. Br Med J 315:629-634. https://doi.org/10.1136/bmj.315.7109.629

13. Heise T, Hermanski L, Nosek L, et al (2012) Insulin degludec: four times lower pharmacodynamic variability than insulin glargine under steady-state conditions in type 1 diabetes. Diabetes, Obes Metab 14:859-864. https://doi.org/10.1111/j.1463-1326.2012.01627.x

14. Higgins J, Li T, Deeks J (2019a) Chapter 6: choosing effect measures and computing estimates of effect. In: Higgins J, Thomas J, Chandler J, et al. (eds) Cochrane Handbook for Systematic Reviews of Interventions version 6.0. Cochrane

15. Higgins J, Savović J, Page M, et al (2019b) Chapter 8: Assessing risk of bias in a randomized trial. In: Higgins J, Thomas J, Chandler J, et al. (eds) Cochrane Handbook for Systematic Reviews of Interventions version 6.0. Cochrane

16. Higgins JPT, Jackson D, Barrett JK, et al (2012) Consistency and inconsistency in network metaanalysis: concepts and models for multi-arm studies. Res Synth Methods 3:98-110. https://doi.org/10.1002/jrsm.1044

17. Hutton B, Salanti G, Caldwell DM, et al (2015) The PRISMA extension statement for reporting of systematic reviews incorporating network meta-analyses of health care interventions: checklist and explanations. Ann Intern Med 162:777. https://doi.org/10.7326/M14-2385 
18. International Hypoglycaemia Study Group (2017) Glucose concentrations of less than $3.0 \mathrm{mmol} / \mathrm{l}$ $(54 \mathrm{mg} / \mathrm{dl}$ ) should be reported in clinical trials: a joint position statement of the American Diabetes Association and the Europian Association for the Study of Diabetes. Diabetologia 60:3-6. https://doi.org/10.1007/s00125-016-4146-6

19. Laranjeira FO, de Andrade KRC, Figueiredo ACMG, et al (2018) Long-acting insulin analogues for type 1 diabetes: An overview of systematic reviews and meta-analysis of randomized controlled trials. PLoS One 13:. https://doi.org/10.1371/journal.pone.0194801

20. Liu W, Yang X, Huang J (2018) Efficacy and safety of insulin degludec versus insulin glargine: a systematic review and meta-analysis of fifteen clinical trials. Int J Endocrinol 2018:1-10. https://doi.org/10.1155/2018/8726046

21. Mathieu C, Gillard P, Benhalima K (2017) Insulin analogues in type 1 diabetes mellitus: getting better all the time. Nat Rev Endocrinol 13:385-399. https://doi.org/10.1038/nrendo.2017.39

22. Moher D, Liberati A, Tetzlaff J, Altman DG (2009) Preferred reporting items for systematic reviews and meta-analyses: the PRISMA statement. PLoS Med 6:e1000097. https://doi.org/10.1371/journal.pmed.1000097

23. NICE (National Institute for Health and Care Excellence) (2015) Type 1 diabetes in adults: diagnosis and management (NICE guideline). In: NICE Guidel. [NG17]. https://www.nice.org.uk/guidance/ng17

24. Nikolakopoulou A, Higgins JPT, Papakonstantinou T, et al (2020) CINeMA: an approach for assessing confidence in the results of a network meta-analysis. PLOS Med 17:e1003082. https://doi.org/10.1371/journal.pmed.1003082

25. Pieber TR, Treichel H-C, Hompesch B, et al (2007) Comparison of insulin detemir and insulin glargine in subjects with Type 1 diabetes using intensive insulin therapy. Diabet Med 24:635-642. https://doi.org/10.1111/j.1464-5491.2007.02113.x

26. Puhan MA, Schunemann HJ, Murad MH, et al (2014) A GRADE Working Group approach for rating the quality of treatment effect estimates from network meta-analysis. BMJ 349:g5630-g5630. https://doi.org/10.1136/bmj.g5630

27. Purnell JQ, Hokanson JE, Marcovina SM, et al (1998) Effect of excessive weight gain with intensive therapy of type 1 diabetes on lipid levels and blood pressure: results from the DCCT. Diabetes Control and Complications Trial. JAMA 280:140-6. https://doi.org/10.1001/jama.280.2.140

28. Ratner RE, Gough SCL, Mathieu C, et al (2013) Hypoglycaemia risk with insulin degludec compared with insulin glargine in type 2 and type 1 diabetes: a pre-planned meta-analysis of phase 3 trials. Diabetes, Obes Metab 15:175-184. https://doi.org/10.1111/dom.12032

29. Russell-Jones D, Khan R (2007) Insulin-associated weight gain in diabetes - causes, effects and coping strategies. Diabetes, Obes Metab 9:799-812. https://doi.org/10.1111/j.14631326.2006.00686.x

30. Shea BJ, Reeves BC, Wells G, et al (2017) AMSTAR 2: a critical appraisal tool for systematic reviews that include randomised or non-randomised studies of healthcare interventions, or both. BMJ 358:j4008. https://doi.org/10.1136/bmj.j4008 
31. Tricco AC, Ashoor HM, Antony J, et al (2021) Comparative efficacy and safety of ultra-long-Acting, long-acting, Intermediate-acting, and biosimilar insulins for type 1 diabetes mellitus: a systematic review and network meta-analysis. J Gen Intern Med. https://doi.org/10.1007/s11606-021-06642-7

32. van Valkenhoef $\mathrm{G}$ et. al. (2014) Modeling inconsistency as heterogeneity in network meta-analysis. Draft Manuscr. 2

33. van Valkenhoef G, Tervonen T, de Brock B, Hillege H (2012) Algorithmic parameterization of mixed treatment comparisons. Stat Comput 22:1099-1111. https://doi.org/10.1007/s11222-011-9281-9

34. Vora J, Christensen T, Rana A, Bain SC (2014) Insulin degludec versus insulin glargine in type 1 and type 2 diabetes mellitus: a meta-analysis of endpoints in phase $3 a$ trials. Diabetes Ther 5:435-446. https://doi.org/10.1007/s13300-014-0076-9

\section{Figures}

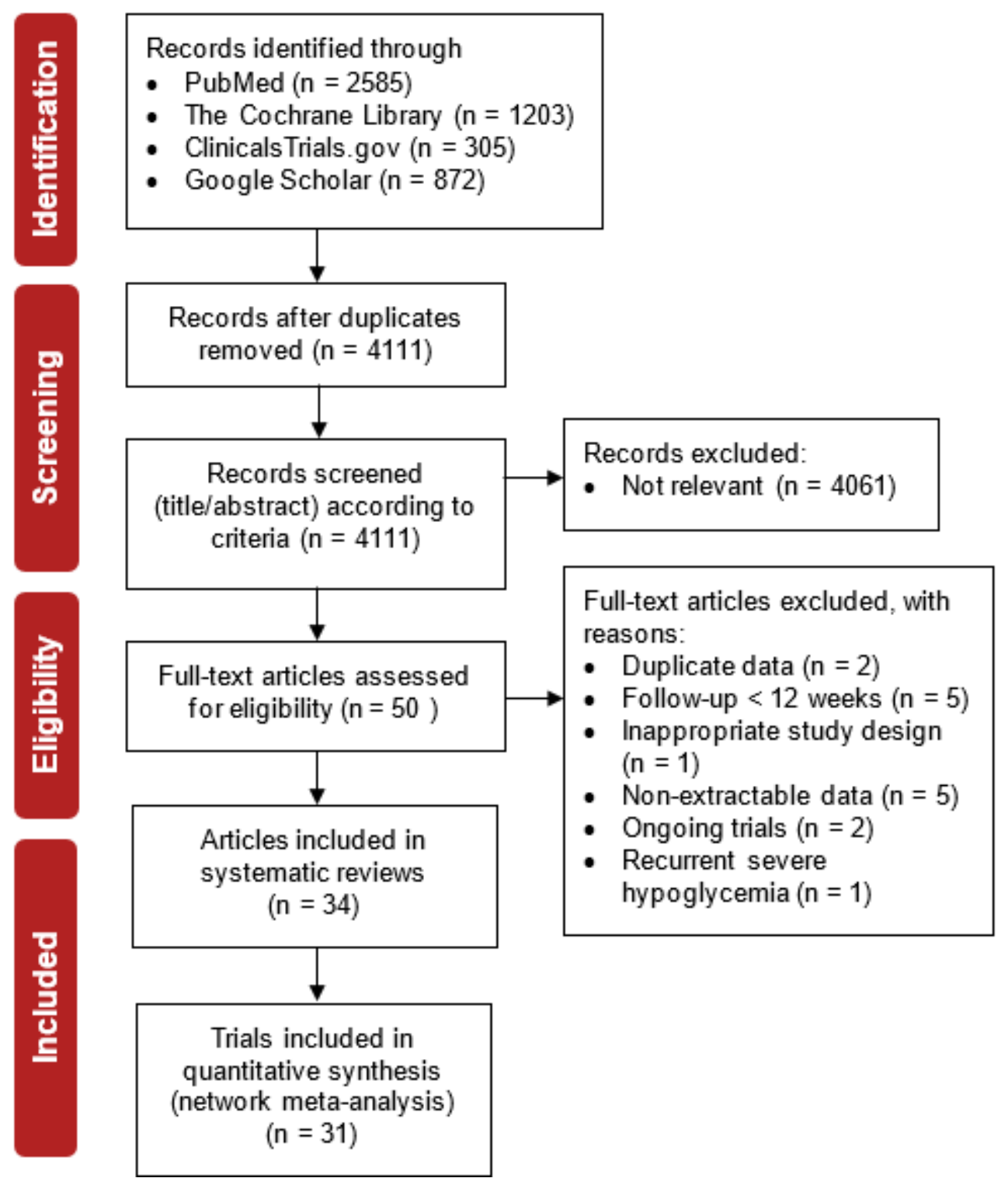

Figure 1 
PRISMA flow diagram of the process for selecting studies with reasons
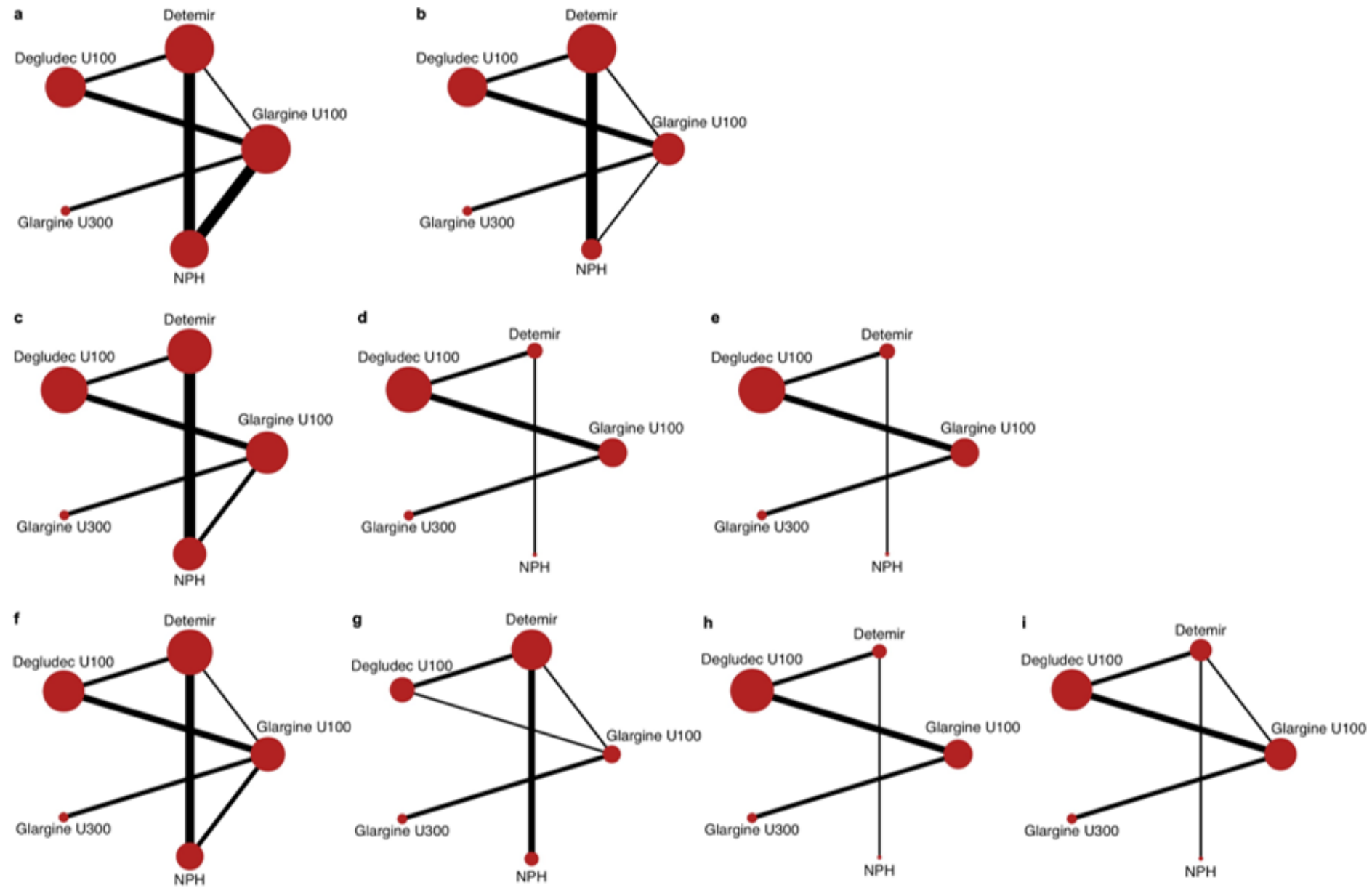

Figure 2

Network graphs of basal insulin treatments (main analysis): a change in $\mathrm{HbA} 1 \mathrm{c}, \mathrm{b}$ change in body weight, $c$ number of patients on severe hypoglycemia, $d$ number of patients on confirmed hypoglycemia, $e$ number of patients on nocturnal confirmed hypoglycemia, $f$ number of events on severe hypoglycemia $g$ number of events on nocturnal severe hypoglycemia, h number of events on confirmed hypoglycemia, and i number of events on nocturnal confirmed hypoglycemia. Each node represents different type of basal insulin and is proportional to the size of randomized participants. Width of lines is proportional to number of trials comparing respective nodes.

\section{Change in $\mathrm{HbA}_{1 \mathrm{c}}(\%)$}

(Relative to Degludec U100)

$\mathrm{NPH}$

Glargine U100

Detemir

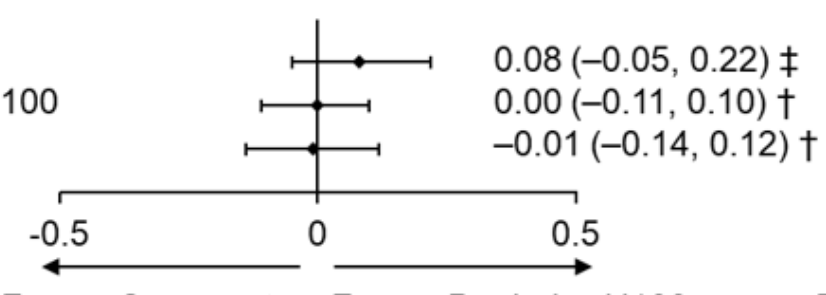

(Relative to Glargine U300)

MD (95\% Crl)

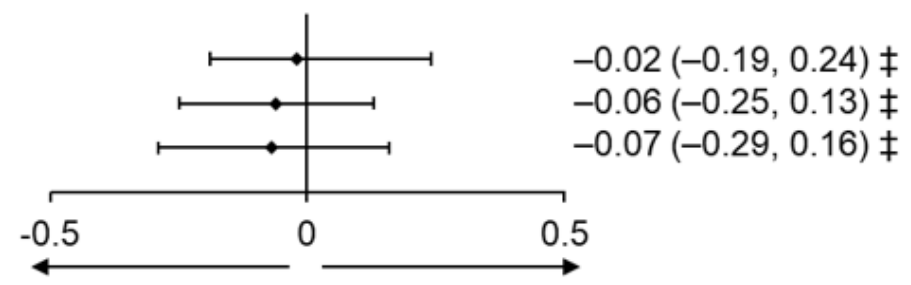

Favors Comparator Favors Degludec U100

Favors Comparator Favors Glargine U300 


\section{Figure 3}

Forest plots of network meta-analysis for change in HbA1c. The GRADE confidence of evidence network was incorporated in this figure: † High certainty evidence and $\ddagger$ Moderate certainty evidence. Crl Credible interval; MD Mean difference.

\section{Severe Hypoglycemia}

(Relative to Degludec U100)

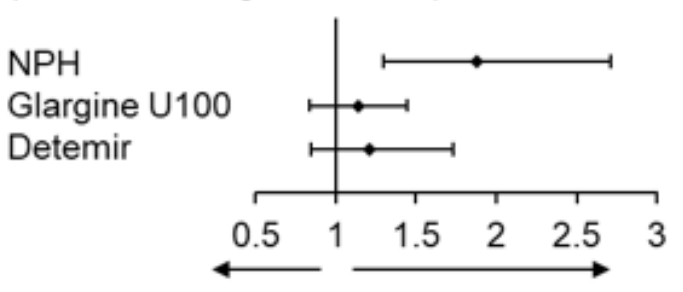

Favors Comparator Favors Degludec U100

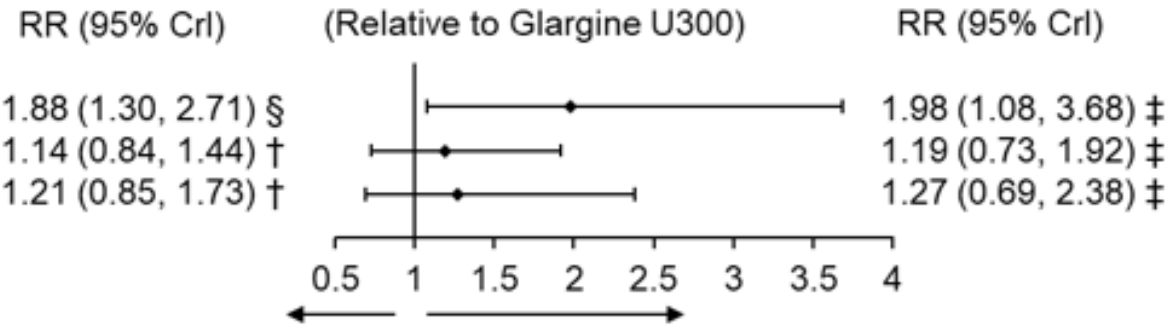

Favors Comparator Favors Glargine U300

\section{Confirmed Hypoglycemia}

(Relative to Degludec U100)

$\mathrm{RR}(95 \% \mathrm{Crl})$

$\mathrm{NPH}$

Glargine U100

Detemir

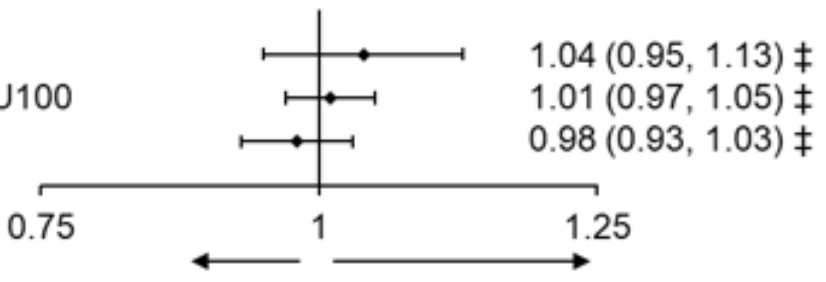

Favors Comparator Favors Degludec U100
(Relative to Glargine U300)

$\mathrm{RR}(95 \% \mathrm{Crl})$

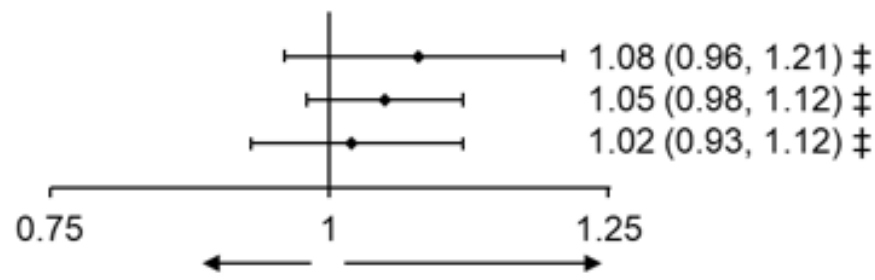

Favors Comparator Favors Glargine U300

\section{Nocturnal Confirmed Hypoglycemia}

(Relative to Degludec U100)

RR (95\% Crl) (Relative to Glargine U300)

$\operatorname{RR}(95 \% \mathrm{Crl})$
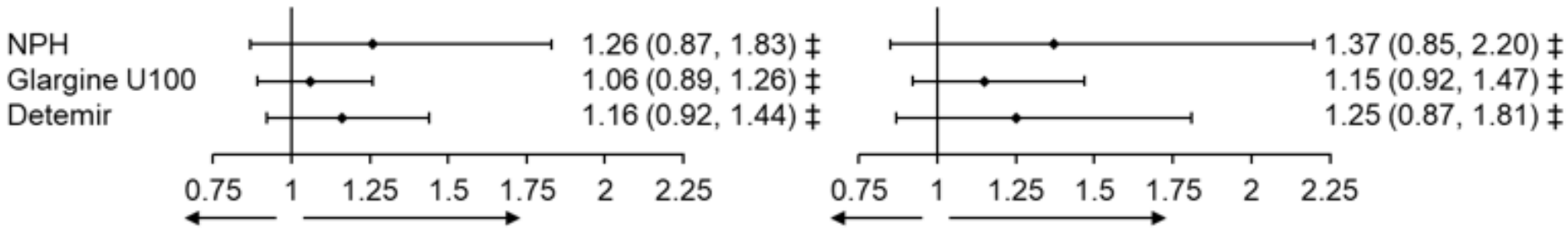

Favors Comparator Favors Degludec U100

Favors Comparator Favors Glargine U300

\section{Figure 4}

Forest plots of network meta-analysis for number of patients on hypoglycemia. The GRADE confidence of evidence network was incorporated in this figure: † High certainty evidence, $\ddagger$ Moderate certainty evidence, and § Low certainty evidence. Crl Credible interval; RR Risk ratio. 


\section{Severe Hypoglycemia}

(Relative to Degludec U100)

$\mathrm{HR}(95 \% \mathrm{Crl})$

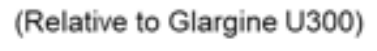

$\operatorname{HR}(95 \% \mathrm{Crl})$

\section{$\mathrm{NPH}$}

Glargine U100

Detemir

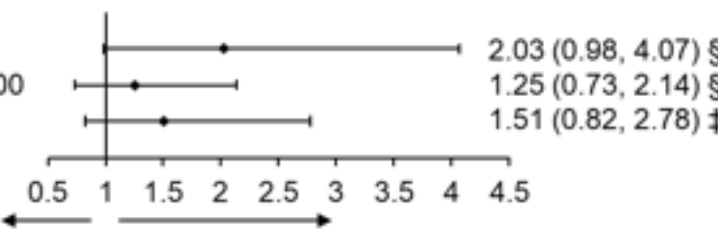

Favors Comparator Favors Degludec U100

Favors Comparator Favors Glargine U300

\section{Nocturnal Severe Hypoglycemia}

(Relative to Degludec U100)

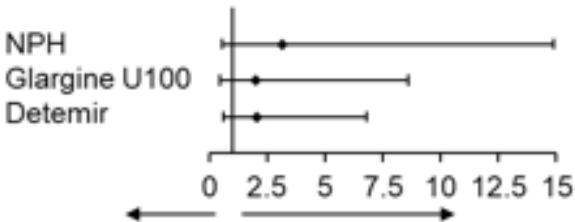

Favors Comparator Favors Degludec U100

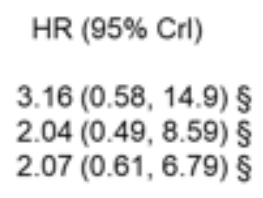
.

Favors Comparator Favors Glargine U300

\section{Confirmed Hypoglycemia}

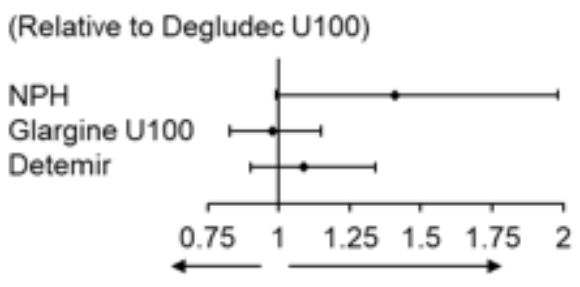

Favors Comparator Favors Degludec U100

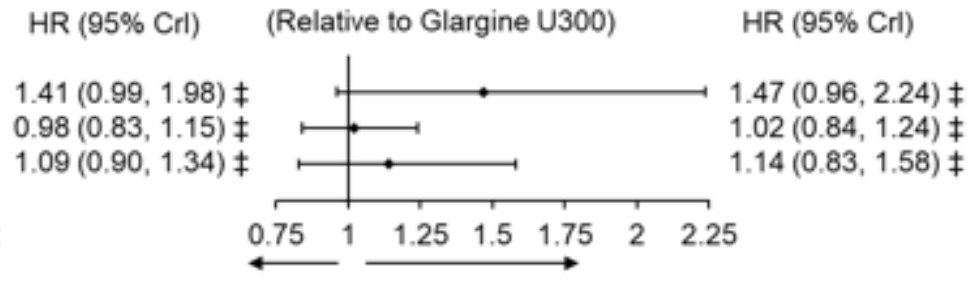

Favors Comparator Favors Glargine U300

\section{Nocturnal Confirmed Hypoglycemia}

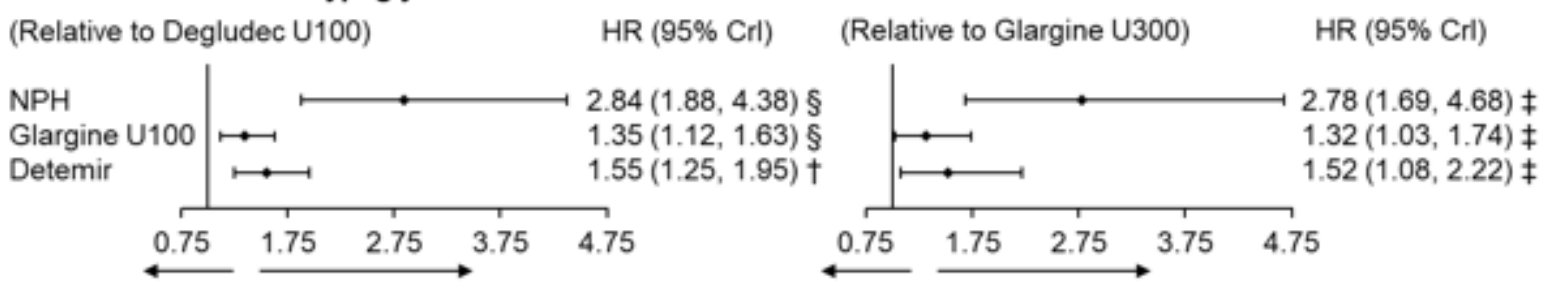

Favors Comparator Favors Degludec U100

Favors Comparator Favors Glargine U300

\section{Figure 5}

Forest plots of network meta-analysis for number of events on hypoglycemia. The GRADE confidence of evidence network was incorporated in this figure: † High certainty evidence, ¥ Moderate certainty evidence, and § Low certainty evidence. Crl Credible interval; HR Hazard ratio. 


\section{Change in Body Weight (kg)}

(Relative to Degludec U100)

$\mathrm{MD}(95 \% \mathrm{Crl})$

(Relative to Glargine U300)

$\mathrm{MD}(95 \% \mathrm{Crl})$

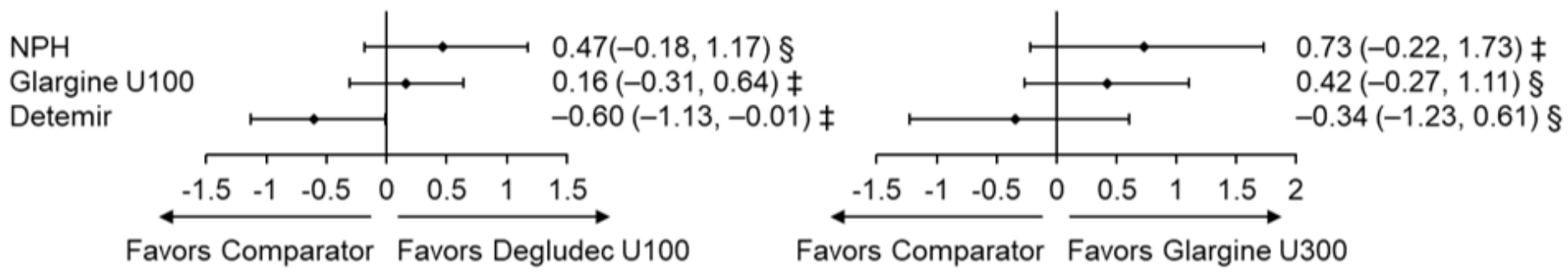

\section{Figure 6}

Forest plots of network meta-analysis for changes in body weight. The GRADE confidence of evidence network was incorporated in this figure: $¥$ Moderate certainty evidence and $\S$ Low certainty evidence. Crl Credible interval; MD Mean difference.

\section{Supplementary Files}

This is a list of supplementary files associated with this preprint. Click to download.

- SupplementalMaterialNSAP.pdf 